\title{
Modeling of a Giant Exoring System Around the Substellar Companion J1407b
}

\author{
Matthew A. Kenworthy ${ }^{1}$ and Eric E. Mamajek ${ }^{2}$ \\ ${ }^{1}$ Leiden Observatory, Leiden University, Niels Bohrweg 2, 2333 RA, Leiden, The Netherlands \\ email: kenworthy@strw.leidenuniv.nl \\ ${ }^{2}$ University of Rochester \\ email: emamajek@pas.rochester.edu
}

\begin{abstract}
We model the complex light curve of 1SWASP J140747.93-394542.6, a 16 Myr old star in the Sco-Cen OB association, with a giant ring system that fills a significant fraction of the Hill sphere of an unseen secondary companion, J1407b. The best ring model has 37 rings and extends out to a radius of $0.6 \mathrm{AU}$ (90 million $\mathrm{km}$ ), and the rings have an estimated total mass on the order of $1 M_{\oplus}$. The ring system has one clearly defined gap at $0.4 \mathrm{AU}$ (61 million $\mathrm{km}$ ), which we hypothesize is being cleared out by a $<0.8 M_{\oplus}$ exosatellite orbiting around the secondary companion.
\end{abstract}

Keywords. techniques: photometric, planetary systems: formation, planets: rings

\section{Introduction}

Extended month- to year-long eclipses indicate the presence of long lived dark disks around secondary companions, including $\epsilon$ Aurigae (Guinan \& Dewarf 2002; Kloppenborg et al. 2010), EE Cep (Mikolajewski \& Graczyk 1999; Graczyk et al. 2003; Mikolajewski et al. 2005), a precessing circumbinary disk around KH 15D (Hamilton et al. 2005; Winn et al. 2006) and three systems recently discovered in the OGLE database - OGLELMC-ECL-17782 (Graczyk et al. 2011), OGLE-LMC-ECL-11893 (Dong et al. 2014) and OGLE-BLG182.1.162852 (Rattenbury et al. 2014).

1SWASP J140747.93-394542.6 (hereafter J1407) is a pre-main sequence 16 Myr old star, $0.9 M_{\odot}, V=12.3 \mathrm{mag} \mathrm{K} 5$ star at $133 \mathrm{pc}$ associated with the Sco-Cen OB Association (Mamajek et al. 2012; van Werkhoven et al. 2014; Kenworthy et al. 2015). The Super Wide Angle Search for Planets (SuperWASP) database (Butters et al. 2010) showed that the star underwent a complex series of eclipses lasting $\sim 56$ days around May 2007 and including a dimming of $>95 \%$. Mamajek et al. (2012) and van Werkhoven et al. (2014) propose that these eclipses are caused by a large ring system orbiting an unseen substellar companion, dubbed J1407b.

Fitting is performed in a two step process - we first constrain the orientation of the ring system using the gradients measured from the light curve, and then use these parameters to generate a model of the ring transmission as a function of radius from the secondary companion.

The light curve $I(t)$ of a source with finite angular size (i.e. the stellar disk of the primary) behind a tilted ring system is not time symmetric (see Figure 1). For each ring boundary, the gradient of the light curve is dependent on both the size of the star, and angle between the local tangent of the ring edge and the direction of motion. Ring structures smaller than that of the stellar diameter are smeared out by the resultant convolution with the stellar disk. 


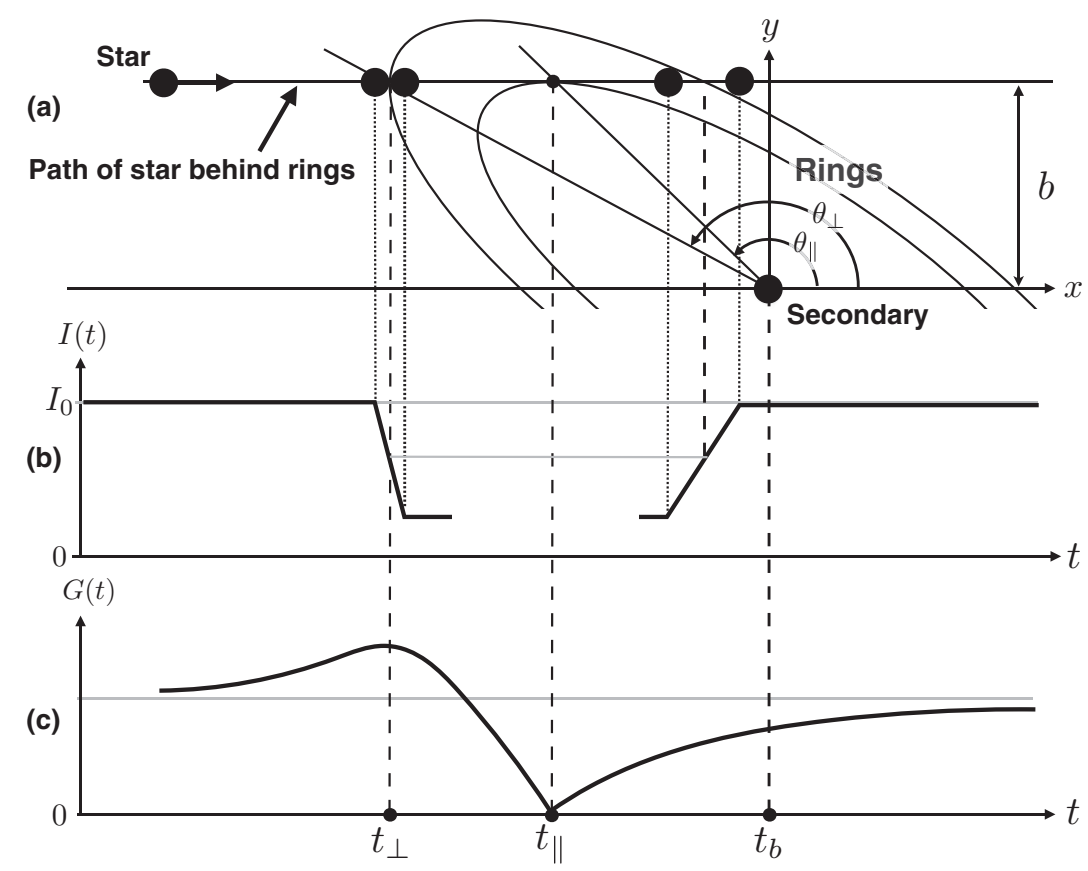

Figure 1. The geometry of the ring model. Panel (a) shows a ring system inclined at an angle of $i_{\text {disk }}$ and rotated from the line of relative velocity by $\phi_{\text {disk }}$. The star passes behind the ring system with impact parameter $b$ at time $t_{b}$. Panel (b) shows the resultant light curve $I(t)$ of the star as a function of time, demonstrating how the local ring tangent convolved with the finite sized disk of the star produces light curves with different local slopes. Panel (c) highlights the three significant epochs in the rate of change of ring radius $r ; t_{b}$ at closest projected separation of the star and the secondary, $t_{\perp}$ where the ring tangent is perpendicular to the direction of stellar motion, and $t_{\|}$where stellar motion is tangent to the ring. $t_{\|}$also marks where the stellar path touches the smallest ring radius.

For a given set of ring orientation parameters $i_{d i s k}, \phi_{d i s k}, b, t_{b}$, we can determine the radial distances of the rings from the secondary companion (i.e. the ring radius) at any epoch, $r(t)=f\left(i_{d i s k}, \phi_{d i s k}, b, t_{b}, t\right)$, and also determine $d r(t) / d t$. The transmission of the disk as a function of $r$ is given by $\tau(r)$. Together with a model of the stellar disk that includes limb darkening (see van Werkhoven et al. 2014) and the functional form of $\tau(r)$, we can calculate the light curve of a ring system model for any epoch.

To understand our ring orientation algorithm, consider a ring system made up of alternately transparent and opaque rings whose radial width would allow complete obscuration or transmission of the stellar disk. The transmitted intensity goes from $I=I_{0}$ to $I=0$ and vice versa at a rate determined by the ring velocity $v$ and the local tangent of the ring to the line of stellar motion, defined as parallel to the x-axis in our model. If the gradient of the light curve is measured close to the midpoint of the transit of a ring edge, and this quantity is plotted as a function of time, the result is the black curve in Figure 1.

The function $G(t)$ represents the maximum flux change possible between a fully transparent and fully opaque ring. For rings that have intermediate values of transmission, the resultant light gradients will lie underneath this black curve, and so the curve represents an upper bound on the light gradient for a given ring orientation. Since we do not know $\tau(r)$, we can use $G(t)$ as an upper limit and we search for ring orientations that have all measured gradients lie underneath this curve. We calculate a cost function 

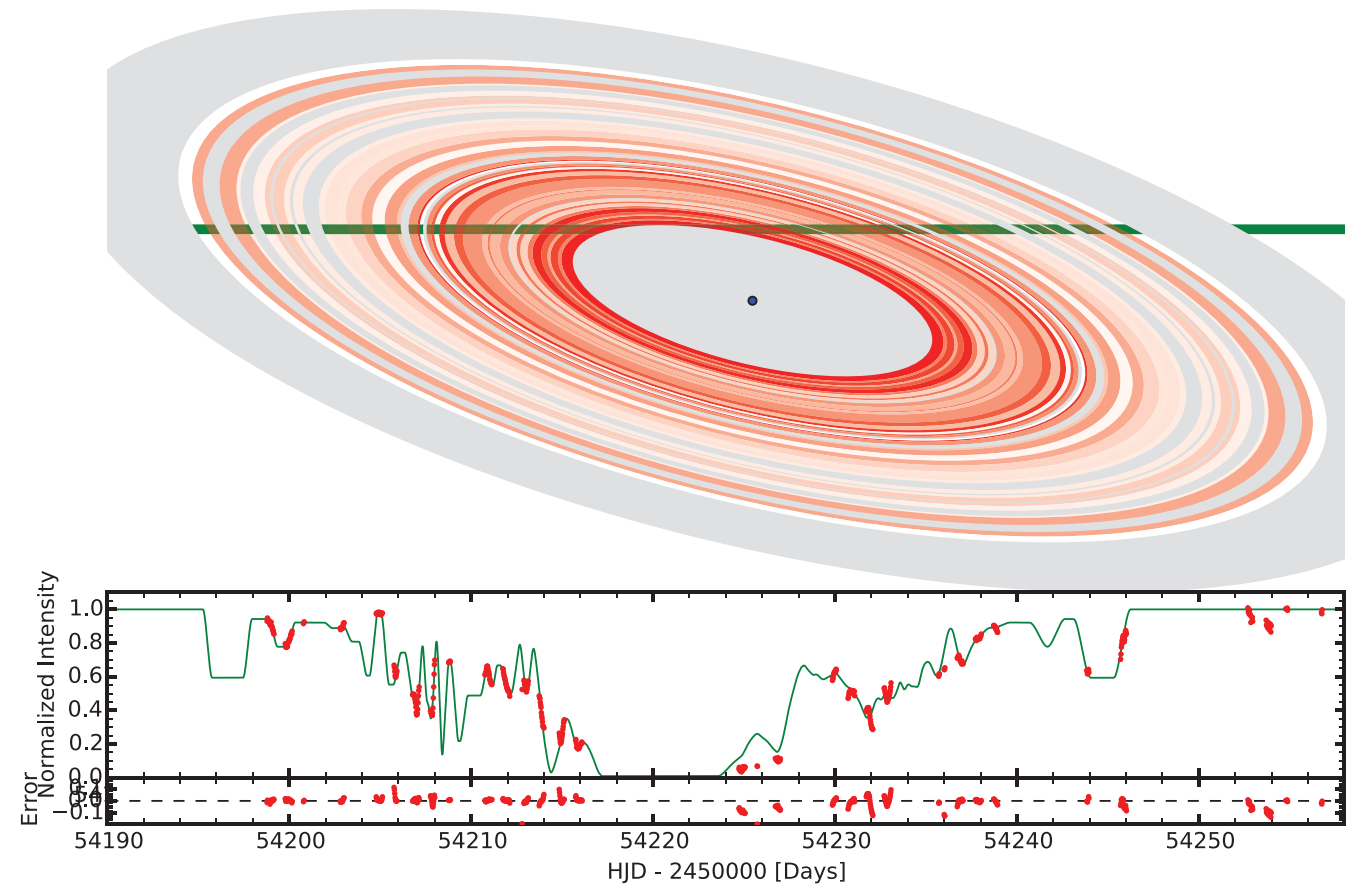

Figure 2. Model ring fit to J1407 data. The image of the ring system around J1407b is shown as a series of nested red rings. The intensity of the colour corresponds to the transmission of the ring. The green line shows the path and diameter of the star J1407 behind the ring system. The grey rings denote where no photometric data constrain the model fit. The lower graph shows the model transmitted intensity $I(t)$ as a function of HJD. The red points are the binned measured flux from J1407 normalised to unity outside the eclipse. Error bars in the photometry are shown as vertical red bars.

that minimises the difference between the model and measured gradients, and penalizes heavily if the measured point goes above the model point. Once the parameters for the disk are determined, we then fit for the ring transmission as a function of radius.

The number of ring edges in the light curve are estimated by counting the number of slope changes identified in the light curve and indirectly implied by the change of the light curve during daylight hours. At least 24 ring edges are required for the number of gradient changes detected in the J1407 data (van Werkhoven et al. 2014), but given the sparseness of the photometric coverage this number is almost certainly higher.

Figure 2 shows one possible ring solution to the J1407 photometric data, where the central eclipse is set at $t_{b}=54220.65$ MJD.

There are clear gaps in all the ring model solutions explored. Gaps in the rings of Solar system giant planets are either caused directly by the gravitational clearing of a satellite or indirectly by a Lindblad resonance due to a satellite on a larger orbit. The J1407 ring system is larger than its Roche limit for the secondary companion. We take the most probable mass and period for the moderate range of eccentricities with mass $23.8 M_{\text {Jup }}$ and orbital period $13.3 \mathrm{yr}$. Gaps in the ring system are either seen directly as the photometric flux from J1407 returning to full transmission during the eclipse, or indirectly as a fit of the model to intermediate transmission photometric gradients. One ring gap with photometry is at HJD 54210, seen during the ingress of J1407 behind the ring system. The corresponding radius for this gap in the disk is seen from 59 million $\mathrm{km}$ to 63 million $\mathrm{km}$ (indicated in Figure 3). If we assume that the gap is equal to the 


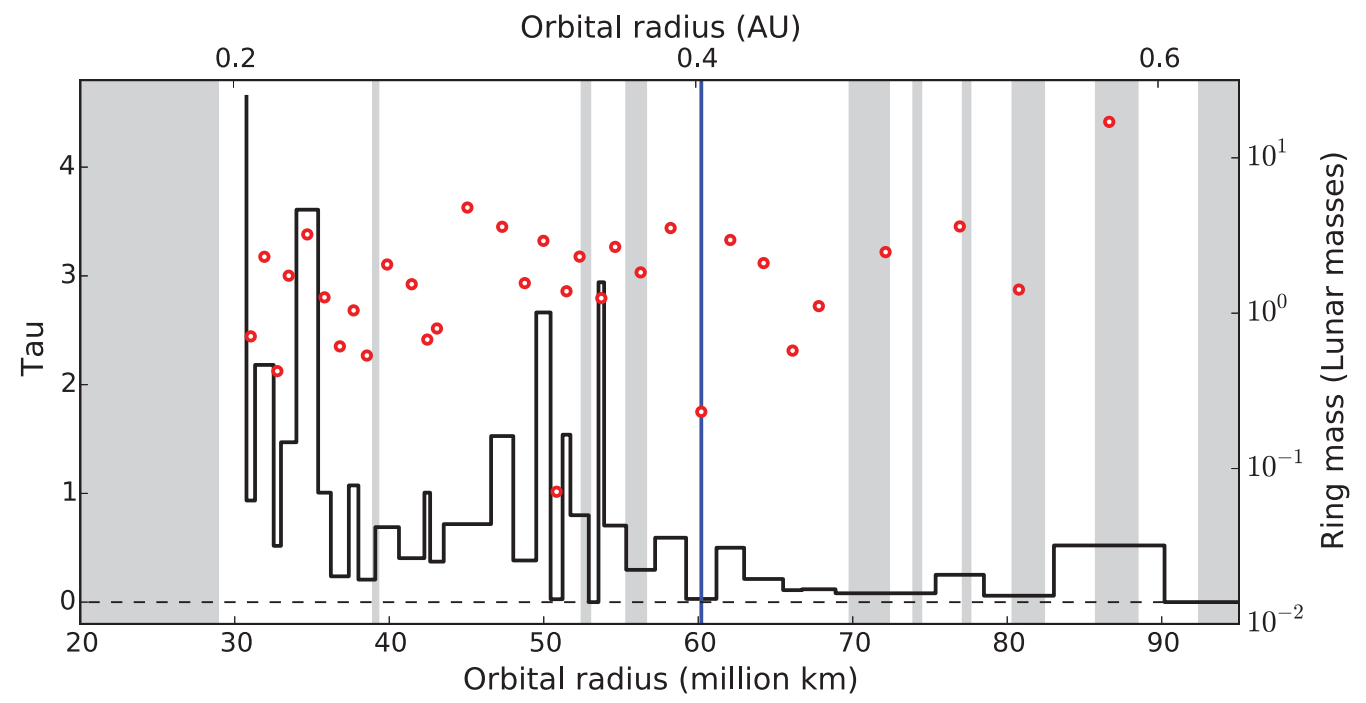

Figure 3. The transmission of the ring model as a function of radius. The grey regions indicate where there in no photometry to constrain the model. The blue line indicates the ring gap seen at 61 million $\mathrm{km}$. Red dots indicate the estimated mass of each ring assuming a mass surface density of $\sim 50 \mathrm{~g} \mathrm{~cm}^{-2}$.

diameter of the Hill sphere of a satellite orbiting around the secondary companion and clearing out the ring, then for the case of $23.8 M_{J u p}$ this corresponds to a satellite mass of $0.8 M_{\oplus}$ and an orbital period of $1.7 \mathrm{yr}$.

With simple assumptions on ring geometry and the ring plane orientation, this ring model reproduces many but not all of the nightly photometric light curves. These discrepancies imply either an error in the determined geometry of the ring plane, and/or the rings are not coplanar.

J1407 is currently being monitored both photometrically and spectroscopically for the start of the next transit. A second transit will enable a wide range of exoring science to be carried out, from transmission spectroscopy of the material, through to Doppler tomography that can resolve ring structure and stellar spot structure significantly smaller than that of the diameter of the star. The orbital period of J1407b is on the order of a decade or possibly longer.

\section{References}

Butters et al. 2010, A\&A , 520, L10

Dong, S., et al. 2014, ApJ , 788, 41

Graczyk, D., Mikołajewski, M., Tomov, T., Kolev, D., \& Iliev, I. 2003, A\&GA , 403, 1089

Graczyk, D. et al. 2011, Acta Astronomica, 61, 103

Guinan, E. F. \& Dewarf, L. E. 2002, in Astronomical Society of the Pacific Conference Series, Vol. 279, Exotic Stars as Challenges to Evolution, ed. C. A. Tout \& W. van Hamme, 121

Hamilton, C. M., et al. 2005, AJ , 130, 1896

Kenworthy, M. A., et al. 2015, MNRAS , 446, 411

Kloppenborg, B., et al. 2010, Nature , 464, 870

Mamajek, E. E., et al. 2012, AJ , 143, 72

Mikolajewski, M., et al. 2005, Ap and $S S, 296,445$

Mikolajewski, M. \& Graczyk, D. 1999, MNRAS , 303, 521

Rattenbury, N. J., et al. 2014, ArXiv e-prints

van Werkhoven, T. I. M., Kenworthy, M. A., \& Mamajek, E. E. 2014, MNRAS , 441, 2845

Winn, J. N., et al. 2006, ApJ , 644, 510 